Pacific Journal of Mathematics

GEVREY CLASSES AND HYPOELLIPTIC BOUNDARY VALUE
PROBLEMS

RATH AmINO 


\title{
GEVREY CLASSES AND HYPOELLIPTIC BOUNDARY VALUE PROBLEMS
}

\author{
RALPh A. ARtino
}

Let $P\left(D, D_{t}\right)$ be a hypoelliptic differential operator with constant coefficients of type $\mu$ with index of hypoellipticity equal to $d \geqq 1$. Let $\Omega$ be an open subset of the half space $R_{+}^{n+1}$ with plane piece of boundary $\omega$ contained in $R_{0}^{n}$. Let $Q_{1}\left(D, D_{t}\right), \cdots, Q_{\mu}\left(D, D_{t}\right)$ be $\mu$ partial differential operators with constant coefficients and consider the boundary value problem:

$$
\begin{aligned}
& P\left(D, D_{t}\right) u=f \text { in } \Omega \\
& \left.Q_{\nu}\left(D, D_{t}\right) u\right|_{\omega}=g_{\nu} \quad 1 \leqq \nu \leqq \mu .
\end{aligned}
$$

In this paper necessary and sufficient conditions are given on $Q_{1}, \cdots, Q_{\mu}$ in order that all solutions $u$ of (1) shall belong to the Gevrey class of index $d$ in $\Omega \cup \omega$ whenever the initial data belong to such classes of functions. In particular, we give not only algebraic conditions but also show how to construct a parametrix for such problems.

Introduction. In 1958 Hörmander first studied regular boundary value problems, giving necessary and sufficient conditions for solutions of (1) to be $C^{\infty}$. (see Hörmander [8]). There, Hörmander gives an algebraic characterization based on the variety of zeros of the characteristic function of the boundary value problem. Later on, it was shown that fundamental solutions to elliptic boundary value problems can be constructed with the aid of this characteristic function (see J. Barros-Neto [4, 5]). Moreover, it can be used to construct a parametrix for hypoelliptic problems (see J. Barros-Neto [6]). In this paper the characteristic function is used to give an algebraic characterization of $d$-hypoelliptic problems. In doing so a different technique is used than that in [8] in order to get more refined estimates. Consequently the special result obtained in [8] for elliptic operators is obtained here. The results here can be extended to Gevrey classes which distinguish the rate of growth of derivatives in different directions. (see [7]). Furthermore, these results have many applications to semi-elliptic problems (see [2]).

The plan of this paper is as follows: In $\S 1 d$-hypoelliptic boundary value problems are defined and the main results are stated. In $\S 2$ the first two equivalences are proved. In $\S 3$ we make use of the parametrix of the boundary value problem and conclude the proof of the main results in $\S 4$.

The author would like to thank Professor Jose Barros-Neto for 
his generous help and suggestions.

1. $d$-hypoelliptic boundary value problems. Let $P\left(D, D_{t}\right)$ be a partial differential operator on $\boldsymbol{R}^{n+1}$ with coefficients in $\boldsymbol{C}$ assumed in the form

$$
P\left(D, D_{t}\right)=D_{t}^{m}+\sum_{j=1}^{m} a_{j}(D) D_{t}^{m-j}
$$

where

$$
D=\left(D_{1}, \cdots, D_{n}\right), \quad D_{j}=\frac{1}{i} \frac{\partial}{\partial x_{j}}, \quad D_{t}=\frac{1}{i} \frac{\partial}{\partial t},
$$

and $a_{j}(D)$ are polynomials in $D$. For any

$$
\begin{aligned}
p & =\left(p_{1}, \cdots, p_{n+1}\right), \quad p_{j} \in N \text { we have } \\
\left(D, D_{t}\right)^{p} & =D_{1}^{p_{1}} \cdots D_{p}^{p_{n}} D_{t}^{p_{n+1}}, \quad|p|=p_{1}+\cdots+p_{n+1} .
\end{aligned}
$$

Definition 1.1. $P\left(D, D_{t}\right)$ is hypoelliptic in $\boldsymbol{R}^{n+1}$ if for some open subset $\mathscr{O} \subset \boldsymbol{R}^{n-1}$ all $u$ in $\mathscr{D}^{\prime}(\mathscr{O})$ such that $P\left(D, D_{t}\right) u=0$ belong to $C^{\infty}(\mathcal{O})$

Definition 1.2. Let $d>0$, by $\Gamma^{d}(\mathcal{O})$ we denote the set of all $u$ in $C^{\infty}(\mathcal{O})$ such that to every compact subset $K \subset \mathcal{O}$, there exists a constant $C(u, K)>0$ such that for all $(n+1)$-tuples of nonnegative integers $p=\left(p_{1}, \cdots, p_{n+1}\right)$,

$$
\operatorname{Sup}_{x \in K}\left|D^{p} u(x)\right| \leqq C^{|p|+1}(|p| !)^{d} .
$$

$\Gamma^{d}(\mathcal{O})$ will be called the Gevrey class of index $d$.

Let $P(\zeta, \tau)$ be the characteristic polynomial of $P\left(D, D_{t}\right)$ and

$$
N=\left\{(\zeta, \tau) \in C^{n} \times C: P(\zeta, \tau)=0\right\} .
$$

The following characterization of hypoelliptic operators was given by Hörmander (see Hörmander [9], or Treves [12]).

THEOREM $(\mathrm{H})$. The following two conditions are equivalent:

(1) $P\left(D, D_{t}\right)$ is hypoelliptic.

(2) If $(\zeta, \tau) \in N,|(\zeta, \tau)| \rightarrow+\infty$ then $|\operatorname{Im}(\zeta, \tau)| \rightarrow+\infty$.

Moreover, given some $d>0$ the following three conditions are equivalent: $\boldsymbol{R}^{n+1}$.

(1) $P\left(D, D_{t}\right) u \in \Gamma^{d}(\mathcal{O})$ implies $u \in \Gamma^{d}(\mathcal{O})$ for all $\mathcal{O}$ open in 
(2) $)_{d}$ There exists a constant $C>0$ such that if $(\zeta, \tau) \in N$ then

$$
|(\zeta, \tau)|^{1 / d}<C(1+|\operatorname{Im}(\zeta, \tau)|) .
$$

(3) $)_{d}$ There exists a $K(x, t)$ in $\Gamma^{d}\left(\boldsymbol{R}^{n+1}-\{0\}\right)$ such that

$$
P\left(D, D_{t}\right) K(x, t)=\delta+\beta
$$

where $\beta$ is analytic in $\boldsymbol{R}^{n+1}$.

The latter conditions imply the former. If (1) or (2) holds, there exists $a d>0$ such that $(1)_{d},(2)_{d}$ and $(3)_{d}$ hold. The set of numbers $d$ for which $(1)_{d},(2)_{d}$ and $(3)_{d}$ hold is a closed half-line $\left[d_{0},+\infty\right)$ with $d_{0}$ rational and $d_{0} \geqq 1$.

The smallest $d$ for which $(1)_{d}$ holds is called the index of hypoellipticity of $P$.

DEFINITION 1.3. A differential operator is of type $\mu$ if there exists a compact set $K \subset \boldsymbol{R}^{n}$ such that whenever $\zeta \in \boldsymbol{R}^{n} \backslash K, P(\zeta, \tau)$, as a polynomial in $\tau$, has $\mu$ roots with positive imaginary parts.

It is easy to prove that for $n>1$ all hypoelliptic operators are of type $\mu$ for some $\mu$. This, however, is not true for $n=1$, as can be seen by the operator associated with:

$P(\zeta, \tau)=\zeta+i \tau$ in $\boldsymbol{R}^{n}$. This is elliptic and hence hypoelliptic but not of any type.

Let $\boldsymbol{R}_{+}^{n+1}=\left\{\left(x_{1}, \cdots, x_{n}, t\right): t>0, x_{i} \in \boldsymbol{R}\right\}$ and $\overline{\boldsymbol{R}}_{+}^{n+1}$ its closure. Let $\Omega$ be an open subset of $\boldsymbol{R}_{+}^{n+1}$ with plane piece of boundary $\omega$ in $\boldsymbol{R}_{0}^{n}=\left\{\left(x_{1}, \cdots, x_{n}, 0\right)\right\}$.

Let $C_{0}^{k}\left(\overline{\boldsymbol{R}}_{+}^{n+1}\right)\left(\right.$ resp. $\left.C_{0}^{k}(\Omega \cup \omega)\right)$ denote the set of $C^{k}$ functions with support in $\overline{\boldsymbol{R}}_{+}^{n+1}(\operatorname{resp} . \Omega \cup \omega)$.

Definition 1.4. Let $Q_{1}\left(D, D_{t}\right), \cdots, Q_{\mu}\left(D, D_{t}\right)$ be $\mu$-partial differential operators with constant coefficients, we say $\left(P\left(D, D_{t}\right), Q_{1}\left(D, D_{t}\right)\right.$, $\left.\cdots, Q_{\mu}\left(D, D_{t}\right)\right)$ defines a $d$-hypoelliptic boundary value problem in $\Omega$ iff:

(1) $P\left(D, D_{t}\right)$ is hypoelliptic of determined type $\mu$ with index of hypoellipticity equal to $d$.

(2) All solutions $u$ in $C^{k}(\Omega \cup \omega)$ of the boundary value problem

$$
\begin{aligned}
& P\left(D, D_{t}\right) u=f \text { in } \Omega \cup \omega \\
& \left.Q_{\nu}\left(D, D_{t}\right) u\right|_{\omega}=g_{\nu} \quad 1 \leqq \nu \leqq \mu,
\end{aligned}
$$

with $f$ in $\Gamma^{d}(\Omega \cup \omega), g_{\nu}$ in $\Gamma^{d}(\omega)$, belong to $\Gamma^{d}(\Omega \cup \omega)$, where $k$ equals the maximum of the orders of $P, Q_{1}, \cdots, Q_{\mu}$. 
Denote by

$$
\mathscr{A}=\left\{\zeta \in C^{n}: P(\zeta, \tau)=0\right.
$$

has $\mu$ roots with positive imaginary parts and none that are real\}.

Let $\tau_{1}(\zeta), \cdots, \tau_{\mu}(\zeta)$ be the $\mu$-roots of $P(\zeta, \tau)$ with positive imaginary parts for each $\zeta$ in $A$. Denote

$$
k_{\zeta}(\tau)=\prod_{\nu=1}^{\mu}\left(\tau-\tau_{\nu}(\zeta)\right)
$$

and

$$
R\left(k_{\zeta}: Q_{1}, \cdots, Q_{\ell^{\prime}}\right)=\frac{\operatorname{det} Q_{l}\left(\zeta, \tau_{k}(\zeta)\right)}{\prod_{l<k}\left(\tau_{k}(\zeta)-\tau_{l}(\zeta)\right)} \equiv C(\zeta)
$$

Definition 1.5. $C(\zeta)$ defined by (1.2) is called the characteristic function of the boundary value problem (1.1).

$C(\zeta)$ is a polynomial in $\zeta, \tau$, and the coefficients of $k_{\zeta}(\tau)$ and $Q_{\nu}(\zeta, \tau) . \quad C(\zeta)$ is defined even in the case of repeated roots. (see Hörmander [8] page 231).

The object of this paper is to prove the following theorem.

THEOREM 1.1. The following conditions are equivalent:

(1) $\left(P\left(D, D_{t}\right), Q_{1}\left(D, D_{t}\right), \cdots, Q_{H}\left(D, D_{t}\right)\right)$ defines a d-hypoelliptic boundary value problem (Definition 1.4).

(2) Every solution $u \in C^{k}(\Omega \cup \omega)$ of the homogeneous boundary value problem

$$
\begin{aligned}
P\left(D, D_{t}\right) u & =0 \text { in } \Omega \cup \omega \\
\lim _{t \rightarrow 0_{+}} Q_{\nu}\left(D, D_{t}\right) u & =0 \text { in } \omega \quad 1 \leqq \nu \leqq \mu .
\end{aligned}
$$

belongs to $\Gamma^{d}(\Omega \cup \omega)$.

(3) Let $C(\zeta)$ be the characteristic function of the boundary value problem $\left(P ; Q_{1}, \cdots, Q_{\mu}\right)$. There exists a constant $M>0$ such that if $\zeta \in C^{n}$ and

$$
|\operatorname{Re} \zeta|^{1 / d} \geqq M(1+|\operatorname{Im} \zeta|)
$$

then $\zeta \in \mathscr{A}$ and $C(\zeta) \neq 0$.

(4) There exist $K_{0}(x, t), K_{1}(x, t), \cdots, K_{\mu^{\prime}}(x, t)$ in $\Gamma^{d}\left(\overrightarrow{\boldsymbol{R}}_{+}^{n+1}-\{0\}\right)$ which satisfy

$$
\left\{\begin{array}{l}
P\left(D, D_{t}\right) K_{0}(x, t)=\delta_{x} \otimes \delta_{t}-\beta(x) \otimes \delta_{t} \text { in } \overline{\boldsymbol{R}}_{+}^{n+1} \\
{\left[Q_{l}\left(D, D_{t}\right) K\right](x, 0)=0 \quad l=1, \cdots, \mu \quad \text { in } \boldsymbol{R}_{0}^{n}}
\end{array}\right.
$$




$$
\begin{cases}P\left(D, D_{t}\right) K(x, t)=0 & \text { in } \overline{\boldsymbol{R}}_{+}^{n+1} \\ Q_{l}\left(D, D_{t}\right) K_{\nu}(x, 0)=\delta_{l, \nu}\left(\delta_{x}-\beta(x)\right) & \text { in } \boldsymbol{R}_{0}^{n}\end{cases}
$$

with $\beta$ analytic in $\boldsymbol{R}^{n}$, and $\delta_{l, \nu}$ is the Kronecker symbol.

2. Proof of Theorem 1.1. That (1) implies (2) is obvious so in this section we prove that (2) implies (3). Denote by $H(\Omega \cup \omega)$ the linear subspace of $C^{k}(\Omega \cup \omega)$ consisting of the solutions of the boundary value problem

$$
\begin{aligned}
& P\left(D, D_{t}\right) u=0 \text { in } \Omega \\
& Q_{\nu}\left(D, D_{t}\right) u=0 \text { in } \omega \quad 1 \leqq \nu \leqq \mu .
\end{aligned}
$$

Call $T_{1}$ the topology on $H(\Omega \cup \omega)$ defined by the semi-norms

$$
\sum_{|p| \leqq k} \sup _{(x, t) \in K}\left|D^{p} u(x, t)\right| \equiv\|u\|_{C^{k}(\Omega \cup \omega), K}
$$

where $K$ runs through the compact subsets of $\Omega \cup \omega . H(\Omega \cup \omega)$ is then a closed linear subspace of $C^{k}(\Omega \cup \omega)$. Let $T_{2}$ be the topology on $H(\Omega \cup \omega)$ defined by the semi-norms

$$
S_{K, \nu}(u)=\sup _{(x, t) \in K} \sum_{p}\left(\frac{1}{|p| !}\right)^{d+1 / \nu}\left|D^{p} u(x, t)\right|
$$

$\nu=1,2, \cdots$ and $K$ runs through the compact subsets of $\Omega \cup \omega$. It is not difficult to show that $T_{2}$ makes $H(\Omega \cup \omega)$ a Frechet space. Now $T_{2}$ is finer than $T_{1}$ hence since any one-to-one continuous linear map of a Frechet space onto another is a homeomorphism, we have that the topologies $T_{1}$ and $T_{2}$ are equivalent. Hence for any compact set $K \subset \Omega \cup \omega$ there is a compact set $H_{\nu}$ and a constant $B_{\nu}>0$ such that for all $u$ in $H(\Omega \cup \omega)$ we have

$$
S_{K, \nu}(u) \leqq B_{\nu}\|u\|_{C^{k}(\Omega \cup \omega), H_{\nu}}
$$

Apply (2.2) to exponential solutions of (2.1),

$$
u(x, t)=e^{i\langle x, t\rangle} v(t) .
$$

Now $u(x, t)$ satisfies (21) if and only if $v(t)$ satisfies

$$
\begin{aligned}
& P\left(\zeta, D_{t}\right) v(t)=0 \\
& Q_{\nu}\left(\zeta, D_{t}\right) v(0)=0, \quad \nu=1,2, \cdots, \mu
\end{aligned}
$$

It follows from Theorem 1.1 page 230 in [8] that (2.3) has a nontrivial solution if and only if $\zeta \in \mathscr{A}$ and $C(\zeta)=0$. We now apply (2.2) taking only derivatives in $t$ up to order $k$ and all orders in $x$ and get 


$$
\begin{aligned}
& \sup _{(x, t) \in K} \sum_{\substack{p^{\prime} \\
l \leqq k}}\left(\frac{1}{\left|p^{\prime}\right| !}\right)^{d+1^{\prime / \nu}}\left|\zeta^{p^{\prime}}\right|\left|e^{i\langle x, \zeta\rangle} v^{(l)}(t)\right| \\
& \quad \leqq B_{\nu} \sup _{(x, t) \in H_{\nu}} \sum_{|p| \leqq k}\left|e^{i\langle x, \zeta\rangle_{\zeta} p^{\prime}}\right|\left|v^{\left\langle p_{n+1}\right)}(t)\right|
\end{aligned}
$$

where $p=\left(p^{\prime}, p_{n+1}\right)$. Now since $\left(p^{\prime}+l\right) ! \leqq p^{\prime} ! l ! 2^{\left(p^{\prime}+l\right)}$ the left-hand side of the last inequality can be replaced by

$$
\sup _{(x, t) \in K} \sum_{\substack{p^{\prime} \\ l \leqq k}}\left(\frac{1}{2^{p^{\prime}} 2^{l} l ! p^{\prime} !}\right)^{d+1 / \nu}\left|\zeta^{p^{\prime}}\right| e^{i\langle x, \zeta\rangle}|| v^{(l)}(t) \mid
$$

and since $l \leqq k$ this in turn can be replaced by

$$
\frac{1}{2^{k} k !} \sum_{p^{\prime}}\left(\frac{1}{\left|p^{\prime}\right| !}\right)^{d+1 / \nu}\left|\left(\frac{\zeta}{2^{d+1}}\right)^{p^{\prime}}\right| \sup _{\langle x, t) \in K} \sum_{l \leqq k}\left|e^{i\langle x, \zeta\rangle} v^{(l)}(t)\right| \text {. }
$$

Using this and (2.2) we get

$$
\begin{aligned}
& \sum_{p^{\prime}}\left(\frac{1}{\left|p^{\prime}\right| !}\right)^{d+1 / \nu}\left(\frac{\zeta}{2^{d+1}}\right)^{p^{\prime}} \sup _{\langle x, t) \in K}\left|e^{i\langle x, \zeta\rangle}\right| \sum_{l \leqq k}\left|v^{l}(t)\right| \\
& \quad \leqq\left(2^{k} k !\right)^{d+1} B_{\nu}|\zeta|^{k} e^{\imath\langle x, \zeta\rangle} \sup _{(x, t) \in H_{\nu}} \sum_{m \leqq k}\left|v^{(m)}(t)\right| .
\end{aligned}
$$

Letting $\delta=\sup _{(x, t) \in K}|x|$ and $\delta_{\nu}=\sup _{(x, t) \in H_{\nu}}|x|$ one gets

$$
\begin{aligned}
& \sum_{p^{\prime}}\left(\frac{1}{\left|p^{\prime}\right| !}\right)^{d+1 / \nu}\left|\left(\frac{\zeta}{2^{d+1}}\right)^{p^{\prime}}\right| \sup _{(x, t) \in K} \sum_{l \leqq k}\left|v^{(l)}(t)\right| \\
& \quad \leqq\left(2^{k} k !\right)^{d+1} B_{\nu} e^{\left(\delta+\delta_{\nu}\right\rangle !|\mathrm{m} \zeta|} \sup _{\langle x, t) \in H_{\nu}} \sum_{m \leqq k}\left|v^{(m)}(t)\right| .
\end{aligned}
$$

It may be assumed that $K \cong H$. Let $a$ be the positive number such that $t$ attains all values between 0 and $a$ when $(x, t) \in K$ and $b$ the number such that $t$ attains all value between 0 and $b$ when $(x, t) \in H_{\nu}$. Thus the last inequality can be written

$$
\begin{aligned}
& \sum_{p^{\prime}}\left(\frac{1}{\left|p^{\prime}\right| !}\right)^{d+1 / \nu}\left|\left(\frac{\zeta}{2^{d+1}}\right)^{p^{\prime}}\right| \sum_{l \leqq l k} \sup _{0 \leqq t \leqq a}\left|v^{(l)}(t)\right| \\
& \quad \leqq\left(2^{k} k !\right)^{d+1} B_{\nu}|\zeta|^{k} e^{\left(\delta+\delta_{\nu}\right)|I m \zeta|} \sum_{m \leqq k} \sup _{0 \leqq t \leqq b}\left|v^{(m)}(t)\right| .
\end{aligned}
$$

Now since $v(t)$ satisfies $(2.3)$ so does $v^{(r)}(t)$ for $0 \leqq r \leqq k$, it follows from [8] pages 234 and 248 that

$$
\sup _{0 \leqq t \leqq b}\left|v^{(r)}(t)\right| \leqq(b / a)^{m-1} \sup _{0 \leqq t \leqq a}\left|v^{(r)}(t)\right| .
$$

Hence there is a constant $B_{\downarrow} \geqq 0$ such that

$$
\sum_{p^{\prime}}\left(\frac{1}{\left|p^{\prime}\right| !}\right)^{d+1 / \nu}\left|\left(\frac{\zeta}{2^{d+1}}\right)^{p^{\prime}}\right| \leqq B_{\nu}^{\prime}|\zeta|^{k} e^{\left(\hat{o}+\delta_{\nu}\right)|\operatorname{Im} \zeta|} .
$$


To simplify notation replace $\zeta$ by $2^{d+1} \zeta$. Hence for suitable constants $\bar{\delta}, \bar{\delta}_{\nu}, C_{\nu}$ we get

$$
\sum_{p^{\prime}}\left(\frac{1}{\left|p^{\prime}\right| !}\right)^{d+1 / \nu}\left|\zeta^{p^{\prime}}\right| \leqq C_{\nu}|\xi|^{k} e^{\left(\bar{\delta}+\bar{\delta}_{\nu}\right)|\operatorname{Im} \xi|} .
$$

Now observe that

$$
|\zeta|^{k} \leqq \sum_{|p|=k}\left(\frac{k !}{p !}\right)^{1 / 2}\left|\zeta^{p}\right| \leqq n^{k / 2} \sum_{|p|=k}\left|\zeta^{p}\right|
$$

Hence (2.4) implies

$$
\sum_{r=0}^{\infty}\left(\frac{1}{r !}\right)^{d+1 / \nu}\left(\frac{|\zeta|}{n^{1 / 2}}\right)^{r} \leqq C_{\nu} e^{\left(\bar{\delta}+\bar{\delta}_{\nu}\right)|\operatorname{Im} \xi|}|\zeta|^{k} .
$$

It follows from this and Lemma 7.4 of [12], that there are constants $\gamma_{2}>0, B_{\nu}^{\prime}>0, D_{\nu}>0$ such that

$$
e^{\gamma_{2}|\xi| 1 /(d+1 / \nu)} \leqq B_{\nu}^{\prime} e^{D_{\nu}|\operatorname{lm} \xi|}|\xi|^{k} .
$$

By taking logs one gets if $\zeta \in \mathscr{A}$ and $C(\zeta)=0$ that there exists a constant $M_{\nu}>0$ such that

$$
|\zeta|^{1 /(d+1 / \nu)} \leqq M_{\nu}(1+|\operatorname{Im} \zeta|+k \log |\zeta|) .
$$

Since $\log |\zeta| /|\zeta|^{s} \rightarrow 0$ as $|\zeta| \rightarrow \infty$ for any $s>0$, the term $k \log |\zeta|$ can be absorbed on the left and a change of constant from this and from the change in variable gives

$$
|\zeta|^{1 /(d+1 / \nu)} \leqq M_{\nu}^{\prime}(1+|\operatorname{Im} \zeta|) \text {. }
$$

LEMMA 2.1 The following conditions are equivalent:

(1) $\zeta \in \mathscr{A}, C(\zeta)=0$ and $|\zeta| \rightarrow+\infty$ implies $|\operatorname{Im} \zeta| \rightarrow+\infty$.

(2) There exist constants $M, \gamma$ both positive such that the set

$$
D=\left\{\zeta \in C^{n}:|\operatorname{Re} \zeta|^{1 / \gamma}>M(1+|\operatorname{Im} \zeta|)\right\}
$$

is contained in $\mathscr{A}$ and $C(\zeta) \neq 0$ for all $\zeta \in D$. Furthermore, the set of all $\gamma$ such that $|\operatorname{Re} \zeta|^{1 / \gamma} \geqq M(1+|\operatorname{Im} \zeta|)$ for $\zeta$ in $\mathscr{A}$ and $C(\zeta) \neq 0$ is a closed half line $\left[\gamma_{0},+\infty\right)$ with $\gamma_{0}$ rational and $\geqq 1$.

Lemma 2.1 is used to complete the proof of (2) implies (3). Since (2.6) implies for $\zeta \in \mathscr{A}$ and $C(\zeta)=0,|\zeta| \rightarrow+\infty$ implies $|\operatorname{Im} \zeta| \rightarrow+\infty$, one can use Lemma 2.1 to take the limit in (2.6) to obtain

$$
|\zeta|^{1 / d} \leqq M(1+|\operatorname{Im} \zeta|)
$$

for some $M>0$. This completes the proof. 
We conclude this section with the proof of Lemma 2.1 which relies on the elimination theorem due to Seidenberg.

Proof of Lemma 2.1. One obviously sees that (2) implies (1). So we prove (1) implies (2). First note that there exist constants $C_{1}>0$ and $\gamma_{1}>0$ both positive such that $\mathscr{A}$ contains all $\zeta \in C^{n}$ such that

$$
|\operatorname{Re} \zeta|^{1 / \gamma_{1}} \leqq C_{1}(1+|\operatorname{Im} \zeta|) .
$$

Indeed, let $\gamma_{1}$ and $C_{1}$ be as in Theorem $(\mathrm{H})$. Let $\tau$ be real, then

$$
|\operatorname{Re}(\zeta, \tau)|^{1 / \gamma_{1}} \geqq|\operatorname{Re} \zeta|^{1 / \gamma_{1}} \geqq M(1+|\operatorname{Im} \zeta|)=M_{1}(1+|\operatorname{Im}(\zeta, \tau)|) .
$$

This implies that $P(\zeta, \tau) \neq 0$. Thus $P(\zeta, \tau)=0$ has no real roots if (2.7) holds. Hence the number of roots of $P(\zeta, \tau)=0$ with positive imaginary parts in constant in each component of the set defined by 2.7 .

Now let $A$ be the set of point in a Euclidean space whose entries are respectively: $\operatorname{Re} \zeta, \operatorname{Im} \zeta, r, t, \operatorname{Re} \tau_{1}(\zeta), \cdots, \operatorname{Re} \tau_{\mu}(\zeta), \operatorname{Im} \tau_{1}(\zeta), \cdots$, $\operatorname{Im} \tau_{\mu}(\zeta)$, the real and imaginary parts of the coefficients of $k_{\xi}(\tau)$, $Q_{1}(\zeta, \tau), \cdots, Q_{\mu}(\zeta, \tau)$. The set of points $A$ satisfies the following set of polynomial equations and inequalities:

$$
\begin{gathered}
t>0,|\operatorname{Im} \zeta|^{2} \leqq \frac{1}{t^{2}}, r>0,|\operatorname{Re} \zeta|^{2}=\frac{1}{r^{2}} \\
P(\zeta, \tau)=\prod_{j=1}^{m}\left(\tau-\tau_{j}(\zeta)\right), \quad k_{\zeta}(\tau)=\prod_{i=1}^{\mu}\left(\tau-\tau_{i}(\zeta)\right) \\
\operatorname{Im} \tau_{1}(\zeta)>0, \cdots, \operatorname{Im} \tau_{\mu}(\zeta)>0, \operatorname{Im} \tau_{\mu+1}(\zeta)<0, \cdots, \operatorname{Im} \tau_{m}(\zeta) \\
C(\zeta)=0 .
\end{gathered}
$$

The set $A$ is thus a semi-algebraic set (see Treves [12]). To $A$ we apply the projection $p: x \in A \rightarrow(\operatorname{Re} \zeta, r, t, \operatorname{Im} \zeta)$. We obtain a set $B$ which is semi-algebraic (Seidenberg-Tarski theorem). In fact, the set $B$ satisfies the equations and inequalities

$$
|\operatorname{Re} \zeta|^{2}=\frac{1}{r^{2}}, \quad|\operatorname{Im} \zeta|^{2} \leqq \frac{1}{t^{2}}, t>0, r>0 .
$$

Assuming (1) in Lemma 2.1, there is a number $r_{0}>0$ such that on the sphere $|\operatorname{Re} \zeta|=1 / r, 0<r<r_{0}$, the function $|\operatorname{Im} \zeta|$ is positive. We set

$$
t(r)=\sup _{r|\operatorname{Re} \zeta|=1}|\operatorname{Im} \zeta|^{-1}, 0<r<r_{0} .
$$

We apply to the set $B$ the projection 


$$
(\operatorname{Re} \zeta, r, t, \operatorname{Im} \zeta) \longrightarrow(r, t) \text {. }
$$

Again using the Seidenberg-Tarski theorem, its image, $C$, is semialgebraic. Now since $|\operatorname{Im} \zeta|^{-1}$ reaches its maximum on $r|\operatorname{Re} \zeta|=1$ for $0<r<r_{0}$, the point $(r, t(r))$ belongs to $C$. In fact, for $0<r_{1}<r_{0}$, $t\left(r_{1}\right)$ is the maximum of $t$ on the intersection of $C$ with the "vertical" line $r=r_{1}$ in the $(r, t)$-plane. This shows that when $r$ varies in an open interval $\left(0, r_{0}\right)$ the point $(r, t(r))$ varies on the boundary of $C$. Observe also that $t(r)$ is a continuous function of $r$ in $\left(0, r_{0}\right)$. Now $C$ being semi-algebraic, is a finite union of sets $C_{j}, j=1, \cdots, k$, each one of these being defined by a finite set $E_{j}$ of polynomial equations and polynomial inequalities. Now for at least one $C_{j}$ containing $(r, t(r))$, the set of equations $E_{j}$ must be nonempty. Otherwise $(r, t(r))$ couldn't lie on the boundary of $C$, but would lie in its interior. So, there is a finite number of polynomials $Q_{1}, \cdots, Q_{s}$ in two variables with real coefficients, such that for every $r$ in $\left(0, r_{0}\right)$ there is a least one index $i, 1 \leqq i \leqq s$, such that

$$
Q_{i}(r, t(r))=0 \text { for } 0<r<r_{0} \text {. }
$$

But the algebraic varieties of zeros $L_{1}, \cdots, L_{s}$ in $R^{2}$ of $Q_{1}, \cdots, Q_{s}$, respectively, can intersect only in a finite number of points (if one does not lie entirely upon the other, in which case we exclude). Since $r \rightarrow(r, t(r))$ is continuous there must be some $r_{0}^{\prime}$ such that $0<r_{0}^{\prime}<r_{0}$ and an index $i_{0}, 1 \leqq i_{0} \leqq s$, such that for $r \leqq r_{0}^{\prime}$ the points $(r, t(r))$ always lie on $L_{i_{0}}$,

$$
\text { i.e. } Q_{i_{0}}(r, t(r))=0 \text { when } 0<r \leqq r_{0}^{\prime} \text {. }
$$

It follows that $t(r)$ has a converging Puiseux expansion in some neighborhood of theorig in in the complex $r$-plane (See Hörmander [8]):

$$
t(r)=a_{0}\left(r^{1 / q}\right)^{k_{0}}+a_{1}\left(r^{1 / q}\right)^{k_{1}}+\cdots
$$

where $q$ is an integer $>0, k_{j}>k_{j-1}$ (a priori $>0, r<0$ ). Since it may assumed $a_{0} \neq 0$ and one can choose the branch of $r^{1 / q}$ which is positive when $r>0$, then one can assume $a_{0}$ is real and positive. Now by hypothesis $r \rightarrow 0$ implies $t(r) \rightarrow 0$. This is possible only if $k_{0}>0$. Then near $r=0$

$$
t(r)=a_{0} r^{k_{0} / q}\left(1+0\left(r^{1 / q}\right)\right) .
$$

This implies

$$
\frac{t(r)}{a_{0} r^{k_{0}^{\prime} q}} \longrightarrow 1 \text { as } r \longrightarrow 0
$$

Hence 


$$
\frac{t(r)}{a_{0} r^{k_{0}^{\prime} q}}-1<\varepsilon \text { for } \varepsilon \text { small and } r \text { small } .
$$

Hence $t(r)<(1+\varepsilon) a_{0} r^{k_{0} / q}$ which implies that

$$
t(r) \leqq C r_{0}^{k_{0} / q} \text { for } r \text { small } .
$$

Taking $r=|\operatorname{Re} \zeta|^{-1}$ with $\zeta \in \mathscr{A}$ and $C(\zeta)=0$ we get

$$
|\operatorname{Re} \zeta| \leqq C|\operatorname{Im} \zeta|^{q / k_{0}}
$$

when $|\operatorname{Re} \zeta|$ is large. Hence for every $\zeta \in \mathscr{A}$ and $C(\zeta)=0$ we obtain

$$
|\operatorname{Re} \zeta| \leqq[M(1+|\operatorname{Im} \zeta|)]^{q / k_{0}}
$$

where $M$ is a suitable constant. Letting $\gamma=\max \left(r_{1}, q / k_{0}\right)$, the assertion of the lemma is proved.

3. In this section that condition 3 in Theorem 1.1 implies 4 is shown.

Now in view of Theorem $(\mathrm{H})$, there exists a constant $M>0$ such that if $|\zeta|>M$ then $P(\zeta, \tau) \neq 0$. Hence one may define

$$
G_{0}(\zeta, t)=(2 \pi)^{-1} \int_{-\infty}^{\infty} \frac{e^{i t \tau}}{P(\zeta, \tau)} d \tau,|\zeta|>M, t>0 .
$$

We see immediately that $G_{0}(\zeta, t)$ satisfies

$$
P\left(\zeta, D_{t}\right) G_{0}(\zeta, t)=\delta .
$$

That is, $G_{0}(\zeta, t)$ is a fundamental solution for the differential operator $P\left(\zeta, D_{t}\right)$ when $|\zeta|>M$. We modify $G_{0}(\zeta, t)$ to get a fundamental solution $G(\zeta, t)$ of $P\left(\zeta, D_{t}\right)$ which satisfies

$$
\begin{aligned}
& P\left(\zeta, D_{t}\right) G(\zeta, t)=\delta \\
& Q_{\nu}\left(\zeta, D_{t}\right) G(\zeta, 0)=0 \quad 1 \leqq \nu \leqq \mu .
\end{aligned}
$$

If one chooses the constant $M>0$ such that $P(\zeta, \tau) \neq 0$ and $C(\zeta) \neq 0$ when $|\zeta|>M$ (which can be done by hypothesis), then using Theorem 1.2 of Hörmander [8] P. 232, $G(\zeta, t)$ is given by

$$
G(\zeta, t)=G_{0}(\zeta, t)-\sum_{\nu=1}^{\mu}\left[Q_{\nu}\left(\zeta, D_{t}\right) G_{0}(\zeta, 0)\right] H_{\nu}(\zeta, t)
$$

where

$$
H_{\nu}(\zeta, t)=\frac{\begin{array}{c}
R\left(k ; Q_{1}\left(\zeta, \tau_{1}(\zeta)\right), \cdots, Q_{\nu-1}\left(\zeta, \tau_{\nu-1}(\zeta)\right), e^{i t_{\nu}(\zeta) t},\right. \\
\left.Q_{\nu+1}\left(\zeta, \tau_{\nu+1}(\zeta)\right), \cdots, Q_{\mu}\left(\zeta, \tau_{\mu}(\zeta)\right)\right)
\end{array}}{C(\zeta)}
$$

and the numerator is the determinant of the $\mu \times \mu$ matrix obtained 
from $\left(Q_{k}\left(\zeta, \tau_{j}(\zeta)\right)\right), k, j=1, \cdots, \mu$ by replacing the $\nu^{\text {th }}$ row by

$$
e^{i \tau_{1}(\zeta) t}, \cdots, e^{i \tau_{\mu}(\zeta) t} \text {. }
$$

We have the following lemma (see Hörmander [8] p. 233).

LEMMA 3.1. If the zeros of $P(\zeta, \tau)$ satisfy the inequalities $|\tau(\zeta)| \leqq C_{0},|\operatorname{Im} \tau(\zeta)| \geqq 1+C_{1}, C_{1}>0$ then we have

$$
\left|D_{t}^{\beta} G_{0}(\zeta, t)\right| \leqq 2^{m+\beta} C_{0}^{\beta} e^{C_{1} t}
$$

for all $t \geqq 0$ and $\beta \geqq 0$.

Lemma 3.2. Suppose that the set

$$
D=\left\{\zeta:|\operatorname{Re} \zeta|^{1 / d} \geqq M(1+|\operatorname{Im} \zeta|)\right\}
$$

is contained in $\mathscr{A}$ and $C(\zeta) \neq 0$ in $D$, then there are constants $M_{1}>0$ and $C_{1}>0$ such that

$$
\frac{1}{|C(\zeta)|} \leqq M_{1}|\zeta|^{C_{1}}, \quad \zeta \in D
$$

Proof. Let $E$ be the set of points $e$ in a real Euclidean space whose entries are: $\operatorname{Re} \zeta_{1}, \cdots, \operatorname{Re} \zeta_{n}, \operatorname{Im} \zeta_{1}, \cdots, \operatorname{Im} \zeta_{n}, r, t, \operatorname{Re} \tau_{1}, \cdots$, $\operatorname{Re} \tau_{m}, \operatorname{Im} \tau_{1}, \cdots, \operatorname{Im} \tau_{m}$, satisfying the following set of equations and inequalities:

$$
\begin{gathered}
P(\zeta, \tau)=\prod_{j=1}^{m}\left(\tau-\tau_{j}(\zeta)\right), \quad k_{\zeta}(\tau)=\prod_{j=1}^{\mu}\left(\tau-\tau_{j}(\zeta)\right) \\
t>0, r>0,\left.|| \operatorname{Re} \zeta\right|^{2}=\frac{1}{r^{2}}, \frac{1}{|C(\zeta)|} \leqq t \\
|\operatorname{Re} \zeta|^{2} \geqq M^{2}(1+|\operatorname{Im} \zeta|)^{2 d} \\
\operatorname{Im} \tau_{1}>0, \cdots, \operatorname{Im} \tau_{\mu}>0 ; \operatorname{Im} \tau_{\mu+1}<0, \cdots, \operatorname{Im} \tau_{m}<0 . \\
C(\zeta)=R\left(k_{\zeta} ; Q_{1}(\zeta, \tau(\zeta)), \cdots, Q_{\mu}(\zeta, \tau(\zeta))\right) .
\end{gathered}
$$

Since one can assume $d$ is rational, $E$ is a semi-algebraic set. (Treves [12]). Since $C(\zeta) \neq 0$ when $\zeta \in D$, there exists an $r_{0}>0$ such that on the sphere $|\operatorname{Re} \zeta|=1 / r$, where $0<r<r_{0}$, the function $1 /|C(\zeta)|$ is strictly positive. Let

$$
t(r)=\sup _{|r \operatorname{Re} \zeta|=1} 1 /|C(\zeta)|
$$

Now suppose $1 /|C(\zeta)|$ is bounded in $D$. There is nothing to prove in this case. So suppose it is unbounded in $D$, then $t(r) \rightarrow+\infty$ as $r \rightarrow 0$.

Applying the projection $E \rightarrow R^{2}$ given by $x \in E \rightarrow(t, r)$, we get 
a semi-algebraic set (Seidenberg-Tarski theorem). Since $r \rightarrow 0$ implies $t(r) \rightarrow \infty$ we proceed as in Lemma 2.1 to show that there exists a rational number $\varepsilon<0$ and a constant $b_{0}>0$ such that

$$
t(r)=b_{0} r^{s}\left(1+0\left(r^{1 / q}\right)\right)
$$

where $q$ is a positive integer. This implies that there exist constants $M_{1}>0$ and $C_{1}>0$ such that

$$
\frac{1}{|C(\zeta)|}<M_{1}|\zeta|^{C_{1}} \text { when } \zeta \in D
$$

LEMmA 3.3. There are constants $C^{\prime}>0$ and $N>0$ such that all the zeros of $P(\zeta, \tau)$ satisfy

$$
|\tau(\zeta)| \leqq C^{\prime}\left(|\zeta|^{N}+1\right)
$$

Proof. $\quad P(\zeta, \tau)=\tau^{m}+a_{\mu_{-1}} \tau^{m-1}+\cdots+a_{0}$, hence the coefficients $a_{j}$ are polynomials in $\tau$ and the zeros satisfy

$$
|\tau(\zeta)| \leqq 1+\sum_{j=0}^{m-1} \alpha_{j}(\zeta)
$$

Lemma 3.4. There are constants $M_{2}, C_{2}, \gamma_{2}$, all positive, such that the functions

$$
D_{t}^{\beta} G(\zeta, t) \text { and } D_{t}^{\beta} H_{\nu}(\zeta, t) \text { of } \zeta \text { are analytic }
$$

in the set $D$ and satisfy

$$
\begin{aligned}
& \left|D_{t}^{\beta} G(\zeta, t)\right| \leqq M_{2}|\zeta| \gamma_{2} e^{-C_{2} t / \zeta \zeta^{1 / d}} \\
& \left|D_{t}^{\beta} H_{\nu}(\zeta, t)\right| \leqq M_{2}|\zeta| \gamma_{2} e^{-\left.C_{2} t \zeta\right|^{1 d} d}
\end{aligned}
$$

for $t \geqq 0,0 \leqq \beta \leqq \sigma-1, \zeta \in D$.

Proof. 1. Since $P$ is $d$-hypoelliptic

$$
|\operatorname{Re}(\zeta, \tau)| \geqq M^{d}(1+|\operatorname{Im}(\zeta, \tau)|)^{d} \geqq C\left(1+|\operatorname{Im}(\zeta, \tau)|^{d}\right)
$$

implies $P(\zeta, \tau) \neq 0$. If $\tau$ is a complex zero of $P(\zeta, \tau)=0$ then

$$
\begin{aligned}
|\operatorname{Re} \zeta| & \leqq|\operatorname{Re}(\zeta, \tau)| \leqq C\left(1+|\operatorname{Im}(\zeta, \tau)|^{d}\right) \\
& \leqq C_{3}\left(1+|\operatorname{Im} \zeta|^{d}+|\operatorname{Im} \tau|^{d}\right) .
\end{aligned}
$$

Hence

$$
C_{3}|\operatorname{Im} \tau|^{d} \geqq|\operatorname{Re} \zeta|-C_{3}\left(1+|\operatorname{Im} \zeta|^{d}\right),
$$

so since one can assume that for all $\zeta \in D$

$$
|\operatorname{Re} \zeta| \geqq 2 C_{3}\left(1+|\operatorname{Im} \zeta|^{d}\right),
$$


for $\zeta \in D$ and $P(\zeta, \tau)=0$ we have

$$
|\operatorname{Im} \tau| \geqq C_{3}|\operatorname{Re} \zeta|^{1 / d} \text {. }
$$

Since $d \geqq 1$ this implies

$$
|\operatorname{Im} \zeta| \geqq C_{3}|\zeta|^{1 / d}
$$

2. Let $F_{1}, \cdots, F_{\mu}$ be analytic functions of a complex variable $\tau$ and define

$$
R\left(P ; F_{1}, \cdots, F_{\mu}\right)=\frac{\operatorname{det} F_{j}\left(\tau_{l}\right)}{\prod_{j<l}\left(\tau_{l}-\tau_{j}\right)},
$$

where $\tau_{1}, \cdots, \tau_{\mu}$ are the roots of $P(\tau, \zeta)$ with positive imaginary parts. It can be shown (Barros-Neto [4], [5], Hörmander [8]), that

$$
\left|R\left(P ; F_{1}, \cdots, F_{\mu}\right)\right| \leqq \prod_{j=1}^{\mu}\left(\sum_{k=0}^{\mu-1} \sup _{x \in K} \frac{F_{j}^{(k)}(x)}{k !}\right),
$$

where $K$ denotes the convex hull of the zeros $\tau_{1}, \cdots, \tau_{\mu}$.

It follows from (3.3) that

$$
D_{t}^{\beta} H_{\nu}(\zeta, t)=\frac{R\left(k_{\zeta} ; Q_{1}(\zeta, \tau(\zeta)), \cdots,(i \tau(\zeta))^{j} e^{i t \tau(\zeta)}, \cdots, Q_{\mu}(\zeta, \tau(\zeta))\right.}{C(\zeta)} .
$$

It follows from 3.7 that:

The numerator is not greater than the sum over $1 \leqq \sigma_{1}<\cdots<$ $\sigma_{\mu} \leqq \mu$ of a product of factors of the form

$$
\frac{d^{k}}{d \tau^{k}}\left((i \tau(\zeta))^{\beta} e^{i t=(\zeta)}\right) \quad 0 \leqq k \leqq \mu-1
$$

and

$$
Q_{\sigma_{j}}^{(r)}(\zeta, \tau(\zeta)), \quad 0 \leqq r \leqq \mu-1 .
$$

By using Leibnitz's formula, Lemmas 3.3 and 3.6, the first is bounded in absolute value by

$$
C|\zeta|^{C_{1}} e^{-t C_{2}}|\zeta|^{1 / d}
$$

where $C$ and $C_{1}$ and constants depending on $\sigma$.

By using Lemma 3.3 the second is easily bounded by a power of $|\zeta|$. Finally using Lemma 3.2 one gets 3.5.

Inequality 3.4 is proved similarly, the only difference is the use of Lemma 3.1 to take care of the term $G_{0}(\zeta, t)$.

Let $\psi(\xi)$ be in $C_{0}^{\infty}\left(R^{n}\right)$ such that $\psi(\xi)=1$ when $|\xi|<M$ and zero 
when $|\xi| \geqq M+1$. In view of Lemma 3.4 the functions

$$
(1-\psi(\xi)) G(\xi, t)
$$

and

$$
(1-\psi(\xi)) H_{\nu}(\xi, t)
$$

define tempered distributions. Hence we define

$$
\begin{aligned}
& K_{0}(x, t)=\mathscr{F}_{\xi}^{-1}(1-\psi(\xi)) G(\xi, t) \\
& K_{\nu}(x, t)=\mathscr{F}_{\xi}^{-1}(1-\psi(\xi)) H_{\nu}(\xi, t),
\end{aligned}
$$

where $\mathscr{F}_{\xi}^{-1}$ denotes the inverse Fourier transformation wit hrespect to the $\xi$-variable only. It is a simple matter to verify that $K_{0}(x, t)$, $K_{\nu}(x, t)$ satisfy 1.3 and 1.4 in the sense of distributions.

To show $K_{2}(x, t) \in \Gamma^{d}\left(\overline{\boldsymbol{R}}_{+}^{n+1}-\{0\}\right)$, one needs the following lemmas.

Lemma 3.5. Let $D=\left\{\zeta \in C^{n}:|\operatorname{Re} \zeta|^{1 / d} \geqq M(1+|\operatorname{Im} \zeta|)\right\}$. For all $\xi \in \boldsymbol{R}^{n}$ such that $|\xi|^{1 / d}>M+1$, there is a constant $C>0$ such that the sphere

$$
S=\left\{\zeta \in C^{n}:|\zeta-\xi|<\rho\right\}
$$

with $\rho=C|\xi|^{1 / d}$ is contained in D. Furthermore, there is a constant $C_{1}>0$ such that, for all $\zeta \in S$, we have $|\zeta| \leqq C_{1}|\xi|$.

Proof. For $\xi \in \boldsymbol{R}^{n}$ such that $|\xi|^{1 / d}>M+1$ let $d(\xi)$ be the distance between $\xi$ and $\partial D$, the boundary of $D$. Clearly, there is a constant $C_{0}>0$ such that $C_{0} \leqq d(\xi)<+\infty$ for all such $\xi$. Let $\zeta_{0}$ be in $\partial D$ such that $d(\xi)=\left|\xi-\zeta_{0}\right|$. Now

$$
\begin{aligned}
|\xi|^{1 / d} & \leqq\left|\zeta_{0}\right|^{1 / d}+\left|\xi-\zeta_{0}\right|^{1 / d} \\
& \leqq M\left(1+\left|\operatorname{Im} \zeta_{0}\right|\right)+\left|\xi-\zeta_{0}\right|^{1 / d} \\
& \leqq M(1+d(\xi))+(1+d(\xi)) \\
& \leqq C_{0}\left(1+\frac{1}{d(\xi)}\right) d(\xi) \leqq A d(\xi) .
\end{aligned}
$$

It suffices to take $C=A^{-1}$ to see $S \subset D$.

Next if $\zeta \in S$ we have:

$$
|\zeta| \leqq|\xi|+C|\xi|^{1 / d}=|\xi|\left(1+\frac{C|\xi|^{1 / d}}{|\xi|}\right) \leqq C_{1}|\xi|
$$

since $|\xi|$ is bounded below for all $\xi \in R^{n}$ such that $|\xi|^{1 / d} \geqq M+1$.

LEMma 3.7. Let $K(\zeta)$ be a such analytic in the set $D$ and satisfy 


$$
\left.|K(\zeta) \leqq A| \zeta\right|^{r}
$$

then there is a constant $B>0$ depending on $M$ and $d$ but independent of $A$ and $\gamma$ such that

$$
\left|D_{\xi}^{q}\left(\xi^{\alpha} K(\xi)\right)\right| \leqq A B^{|\alpha|+|q|+\gamma}|q| !|\xi|^{r+|\alpha|-|q| \mid d}
$$

for all $\alpha, q$, and real $\xi$ such that $|\xi| \geqq M+1$.

Proof. Since $\left|\xi^{\alpha} K(\xi)\right| \leqq A|\xi|^{r+|\alpha|}$ it suffices to prove 3.10 when $\alpha=0$; for the general result follows when one applies this special case to $\zeta^{\alpha} K(\zeta)$.

It follows from Lemma 3.6 that there is a constant $C>0$ such that the sphere

$$
S=\{|\zeta-\xi| \leqq \rho\}, \quad \rho=C|\xi|^{1 / d}
$$

is contained in $D$ if $|\xi|>M+1$, and a constant $C_{1}>0$ such that for all $\zeta$ in $S,|\zeta|<C_{1}|\xi|$. Hence it follows from 3.11 that in $S$.

$$
|K(\zeta)| \leqq A C_{1}^{r}|\xi|^{r}
$$

But then it follows from Cauchy's inequality for derivative of an analytic function in a sphere that

$$
\left|D_{\xi}^{q} K(\xi)\right| \leqq A C_{1}^{r}|\xi|^{\gamma}|q| !\left(C|\xi|^{1 / d}\right)^{-|q|} .
$$

THEOREM 3.1. The kernels $K_{\nu}(x, t)(1 \leqq \nu \leqq \mu)$ belong to $\Gamma^{d}\left(\overline{\boldsymbol{R}}_{+}^{n+1}-\right.$ $\{0\})$.

Proof. One must show to every compact set $K \subset \overline{\boldsymbol{R}}_{+}^{n+1}-\{0\}$, there exists a constant $C\left(K, K_{\nu}\right)>0$ such that for every $p=\left(p_{1}, \cdots, p_{n}\right)$, $\beta=0,1,2$,

$$
\sup _{(x, t) \in K}\left|D_{x}^{p} D_{t}^{\beta} K_{\nu}(x, t)\right| \leqq C^{|p|+\beta+1}(|p|+\beta) !^{d} .
$$

Let $q=\left(q_{1}, \cdots, q_{n}\right)$ be an $n$-tuple of nonnegative integers consider the integral:

$$
x^{q} D_{x}^{p} D_{t}^{\beta} K_{\nu}(x, t)=(2 \pi)^{-n} \int_{R^{n}} e^{i\langle x, \xi\rangle} D_{\xi}^{d}\left[(1-\psi(\xi)) \xi^{p} D_{t}^{\beta} H_{\nu}(\xi, t)\right] d \xi .
$$

This splits into the sum of the following integrals:

$$
\begin{gathered}
T_{0}=(2 \pi)^{-n} \int_{R^{n}} e^{i\langle x, \xi\rangle}[1-\psi(\xi)] D_{\xi}^{q}\left(\xi^{q} D_{t}^{\beta} H_{\nu}(\xi, t)\right) d \xi \\
T_{r, s}=(2 \pi)^{-n} \int_{R^{n}} e^{i\langle x, \xi\rangle} D^{r}(1-\psi(\xi)) D_{\xi}^{s}\left(\xi^{q} D_{t}^{\beta} H_{\nu}(\xi, t)\right) d \xi,
\end{gathered}
$$


where $r+s=q$ and $r>0$.

1. Estimate of $T_{0}$. In view of 3.5 and 3.11 there is a constant $C>0$ such that

$$
\left|D_{\xi}^{q}\left(\xi^{p} D_{t}^{\beta} H_{\nu}(\xi, t)\right)\right| \leqq|q| ! C^{|p|+|q|+\gamma_{2}}|\xi|^{r_{2}+|p|-|q| \mid d},
$$

hence

$$
\left|T_{0}\right| \leqq(2 \pi)^{-n}|q| ! C^{|p|+|q|+\gamma_{2}} \int_{R^{n}}|\xi|^{r_{2}+|p|-|q| / d} d \xi .
$$

The last integral is absolutely convergent when

$$
|q|>d\left(\gamma_{2}+|p|+n\right) \text {. }
$$

Assuming $\gamma_{2}$ is an integer, as one may, and choosing $|q|^{\pi}$ such that

$$
d\left(\gamma_{2}+|p|+n\right)<|q| \leqq d\left(\gamma_{2}+|p|+n\right)+1,
$$

one can use Euler's gamma function,

$$
\Gamma(x)=\int_{0}^{\infty} t^{x-1} e^{-t} d t,
$$

to estimate $|q|$ ! Accordingly, to every $d \geqq 1$ and $x$ an integer and $a$ a constant, it is easy to prove that there is a constant $C>0$ such that

$$
\Gamma(d x+a+1) \leqq c^{|x|+1}(x !)^{d}
$$

with $C$ independent of $x$.

Using this fact and condition 3.14 on $|q|$ one obtains

$$
|q| ! \leqq C_{1}^{\gamma_{2+|p|+n+1}}\left[\left(\gamma_{2}+|p|+n\right) !\right]^{d} .
$$

Since $(a+b) ! \leqq 2^{a+b} a ! b !$,

$$
\left(\gamma_{2}+|p|+n\right) ! \leqq C_{2}^{\beta+|p|+1}\left(\gamma_{2} \beta\right) !|p| ! .
$$

Using this one obtains a constant $C_{4}>0$ such that

$$
|q| ! \leqq C_{4}^{|p|+1}|p| !^{d} .
$$

From 3.12 and 3.16 one gets the following estimate on $T_{0}$

$$
\left|T_{0}\right| \leqq C_{5}^{|p|+1}(|p|) !^{d}
$$

2. Estimate of $T_{r, s^{*}}$ Using 3.11,

$$
\left|D_{\xi}^{s} \xi^{q} D_{t}^{\beta} H_{\nu}(\xi, t)\right| \leqq C^{|p|+s+\gamma_{2}} s !|\xi|^{\gamma_{2}+\left|p_{i}-\right| s|| d} .
$$


Since the support of $D^{r}(1-\psi(\xi))$, which is denoted by $H$, is contained in the set

$$
\{\xi: M \leqq|\xi| \leqq M+1\}
$$

one may let

$$
R=\sup _{\xi \in I I}|\xi|
$$

Then

$$
\left|T_{r, s}\right| \leqq C_{1}^{p++S+\gamma_{2}} R^{r_{2-i} p i-S / d} s ! .
$$

Since $|s|<|q|$, and choosing $|q|$ as in 3.14, there is a constant $C_{6}>0$ such that

$$
\left|T_{r, s}\right| \leqq C_{6}^{p \mid+1}(|p|)^{d}
$$

The estimates 3.17 and 3.18 show that to every compact set $K \subset \overline{\boldsymbol{R}}_{+}^{n+1}$ there exists a constant $C\left(K, K_{\llcorner}\right)>0$ such that for every $n$-tuple $p=\left(p_{1}, \cdots, p_{n}\right), \beta=0,1, \cdots \sigma-1$ there exists $q=\left(q_{1}, \cdots, q_{n}\right)$ such that $|q| \leqq|p| \alpha+\beta$ where $\alpha$ and $\beta$ are independent of $p$ such that

$$
\sup _{(x, t) \in K}\left|x^{q} D_{x}^{p} D_{t}^{\beta} K_{\nu}(x, t)\right| \leqq C^{|p|+1}(|p|)^{d} .
$$

That $K_{\nu}(x, t)$ belongs to $\Gamma\left(\overrightarrow{\boldsymbol{R}}_{+}^{n+1}-\{0\}\right)$ is a consequence of the following lemma.

Lemma 3.8. Let $K$ be a compact subset of $\bar{R}_{+}^{n+1} \backslash\{0\}$ and $K_{\nu}(x, t)$ a solution of $P\left(D, D_{t}\right) K_{2}(x, t)=0$ such that

$$
\sup _{(x, t) \in K}\left|D^{P} D^{\beta} K_{\downarrow}(x, t)\right| \leqq C^{|p|+1}(|p| !)^{d} \text { for all } p,
$$

and $0 \leqq \beta \leqq \sigma-1$, then $K_{\nu}(x, t)$ belongs to $\Gamma^{d}\left(\bar{R}_{+}^{n-1} \backslash\{0)\right.$.

Proof. Since one can write $P\left(D, D_{t}\right)=D_{t}^{\sigma}+\sum_{j=1}^{\sigma-1} P_{j}(D) D_{t}^{j}$ and $P\left(D, D_{t}\right) K_{\nu}(x, t)=0$ one gets

$$
D_{t}^{\tau} K_{\iota}(x, t)=-\sum_{j=1}^{\sigma-1} P_{j}(D) D_{t}^{\jmath} K_{\iota}(x, t), \text { where } P_{\jmath}(D)
$$

are polynomials in $D$ of degree $\sigma-j$. We shall prove that there exists positive constants $C, M$ such that for any compact subset

$$
\begin{gathered}
K \text { of } \bar{R}_{+}^{n+1} \backslash\{0\} \\
\sup _{(x, t) \in K}\left|D_{t}^{\beta} D^{p} K_{\nu}(x, t)\right| \leqq C^{|\beta|+|p|+1} M^{\beta}(|p|+\beta) !^{d},
\end{gathered}
$$


for all $\beta$ and $p$, where $C$ and $M$ depend only on $K$ and $\sigma$ but not on $p$ and $\beta$. Note that (3.21) is obviously true for all $0 \leqq \beta \leqq \sigma-1$ in view of (3.19).

Now assume that (3.21) is true for all $\beta<\sigma+m$ where $m>0$. One must show it is true for $\beta=\sigma+m$. Differentiating (3.20) one obtains

$$
D^{p} D_{t}^{g+m} K_{\nu}(x, t)=-\sum_{j \geqq \sigma-j} P_{j}(D) D^{p} D_{t} K_{\nu}(x, t)
$$

Now

$$
P_{j}(D)=\sum_{|\mu| \leqq \sigma-j} \alpha_{j \mu} D^{\mu} .
$$

Thus one must estimate terms of the form

$$
a_{j \mu} D^{\mu+p} D_{t}^{m-\jmath} K_{\nu}(x, t) \text {, and then sum them . }
$$

In view of the induction hypothesis

$$
\begin{aligned}
& \left|a_{j \mu} D^{\mu+p} D_{t}^{m+j} K_{\nu}(x, t)\right| \\
& \quad \leqq\left|a_{j^{\mu}}\right| C^{|\mu|+|p|+m+j+1} M^{m+j}(|\mu|+|p|+m+j) !^{d} .
\end{aligned}
$$

Since $j \leqq \sigma-1$ and $|\mu| \leqq \sigma-j$, the right side of the least inequality can be estimated by

$$
\left|a_{j^{\mu}}\right| C^{\sigma+|p|+m+1} M^{m+\sigma-1}(\sigma+|p|+m) !^{d}
$$

Assuming $M$ is larger than $\sum_{\mu} \sum_{j}\left|a_{j \mu}\right|$ one has

$$
\sup _{\langle x, t) \in K} \mid D^{p}\left(D_{t}^{\sigma+m} K_{\nu}(x, t) \mid \leqq M C^{\sigma+|p|+m+1} M^{m+\sigma-1}(\sigma+|p|+m) !^{d} .\right.
$$

This implies $K_{\nu}(x, t) \in \Gamma^{d} \bar{R}_{+}^{n+1} \backslash\{0\}$.

Note that $K_{0}(x, t) \in \Gamma^{d}\left(\bar{R}_{+}^{n+1} \backslash\{0\}\right)$ follows in a similar manner. One must consider an extra term which is analytic and theorefore doesn't affect the agrument.

4. We now show that (4) implies (1) in Theorem 1.1. To do so one needs the following lemma.

LeMmA 4.1. Let $V$ be an open subset of $\boldsymbol{R}_{0}^{n}=\left\{(x, 0): x \in \boldsymbol{R}^{n}\right\}$, and $F$ a continuous function in $\boldsymbol{R}_{0}^{n}$ with compact support such that $F \in \Gamma^{d}(V)$, then $K_{\nu}(x, t)^{* \prime} F(x) \in \Gamma^{d}\left(V \times R_{+}\right)$, where $\left(^{*}\right)^{\prime}$ denotes convolution with respect to the x-variable.

Proof. Let $\mathscr{C}$ be a compact subset of $V$ and let $\mathscr{C}_{1}$ be a relatively compact open subset such that $\mathscr{C} \subset \mathscr{C}_{1} \subset \overline{\mathscr{C}}_{1} \subset V$. Let $\alpha \in C_{0}^{\infty}(V)$ be such that $\alpha \equiv 1$ in $\overline{\mathscr{C}}_{1}$. Write 


$$
K_{\nu}^{*} F=K_{\nu}^{* \prime} \alpha F+K_{\nu}^{* \prime}(1-\alpha) F .
$$

For every $p=\left(p_{1}, \cdots, p_{n}\right), \beta=0,1,2, \cdots$, it follows by induction that

$$
\begin{aligned}
& x_{x}^{p} D_{t}^{\beta}\left(K_{\nu}^{* \prime} \alpha F\right) \\
& \quad=D_{t}^{\beta} K_{\nu}^{* \prime} \alpha D^{p} F+\sum_{j=1}^{m} D_{0} \cdots D_{j-1} D_{t}^{\beta} K_{\nu}^{* \prime}\left(D_{j} \alpha\right)\left(D_{j+1} \cdots D_{m+1} F\right),
\end{aligned}
$$

where $D_{0}=D_{m+1}=$ identity operator, $D^{p}=D_{0} D_{1} \cdots D_{m+1}$. Let

$$
\begin{aligned}
& T_{0}=D_{t}^{\beta} K_{\nu}^{* \prime} \alpha D^{p} F \\
& T_{j}=\sum_{j=1}^{m} D_{0} \cdots D_{j-1} D_{t}^{\beta} K_{\nu}^{* \prime}\left(D_{j} \alpha\right)\left(D_{j+1} \cdots D_{m+1} F\right) .
\end{aligned}
$$

Estimate of $T_{0}$ in $\mathscr{C}$. The support of $\alpha D^{p} F$ is some compact set $L$. The values of $K^{*} \alpha D^{p} F$ in $\mathscr{C}_{1}$ depend only on the values of $K_{\nu}$ on the bounded set $\mathscr{C}_{1}-L$, regarded as a distribution in $x$ depending smoothly on $t$. Hence $K_{\nu}$ equals a distribution of finite order, in $x$, in a neighborhood of $\mathscr{C}_{1}-L$. Then

$$
K_{\nu}=\sum_{|q| \leqq l} D^{q} F_{q}(x, t),
$$

where $F_{q}$ are continuous functions with compact support in a neighborhood of $\mathscr{C}_{1}-L \subset \boldsymbol{R}_{0}^{n}$, and belonging to $\Gamma^{d}\left(\overline{\boldsymbol{R}}_{+}\right)$in $t$. Then one can write:

$$
T_{0}=\sum_{\mid q i<l} \int_{V} D_{t}^{\beta} F_{q}(x-y, t) D^{q}\left(\alpha(y) D^{p} F(y)\right) d y .
$$

Hence, after applying Leibnitz's formula,

$$
\sup _{\substack{x \in \mathbb{E} \\ 0 \leqq t \leqq o}}\left|D_{t}^{\beta} K_{\nu}^{* \prime} \alpha D^{p+q} F\right| \leqq C_{1}^{\beta+1}(\beta !)^{d} \sup _{y \in \mathscr{\varepsilon}}\left|\alpha D^{p+q} F(y)\right| \text {, }
$$

$\delta$ is a small number $>0$.

Since $F \in \Gamma^{d}(V)$ one gets

$$
\begin{aligned}
\sup _{\substack{x \in \mathscr{q} \\
0 \leqq t \leqq o}}\left|D_{t}^{\beta} K_{\nu}^{* \prime} \alpha D^{p+q} F\right| & \leqq C_{1}^{\beta+1}(\beta !)^{d} C_{2}^{|p|+|q|+1}(|p|+|q|) !^{d} \\
& \leqq C^{\beta+|p|+1}(|p|+\beta) !^{d} .
\end{aligned}
$$

Estimate of $T_{j}$ in $\mathscr{C}$. Since $\alpha \equiv 1$ in $\overline{\mathscr{C}}_{1}, D_{j} \alpha=0$ in $\overline{\mathscr{C}}_{1}$, hence the support of $\left(D_{j} \alpha\right)\left(D_{j+1} \cdots D_{m+1} F\right)$ is contained in

\section{$\operatorname{Supp} \alpha \cap \overline{\mathscr{C}}_{1}^{C}$,}

where $\overline{\mathscr{C}}_{1}^{c}$ denotes the complement of $\overline{\mathscr{C}}_{1}$ in $\boldsymbol{R}_{0}^{n}$. On the other hand, the values of $\left(D_{0} \cdots D_{j}\right) D_{t}^{\beta} K_{\nu}^{* \prime}\left(D_{j} \alpha\right)\left(D_{j+1} \cdots D_{m+1} f\right)$ in $\mathscr{C}_{1}$ depend on the values of $\left(D_{0} \cdots D_{j}\right) D_{t}^{\beta} K_{\nu}$ on $\mathscr{C}_{1}-\operatorname{supp} \alpha \cap \overline{\mathscr{C}}_{1}^{C}$, a subset of 
$\boldsymbol{R}_{0}^{n}-\{0\}$. Since on this set $K_{\nu}$ belongs to $\Gamma^{d}$ we get

$$
\sup _{\substack{x \in \mathbb{E}_{1} \\ 0 \leqq t \leqq \delta}}\left|D_{0} \cdots D_{j-1} D_{t}^{\beta} K_{\nu}(x, t)\right| \leqq C^{\beta+j}(j+\beta) !^{d} .
$$

Since $F \in \Gamma^{d}(V)$ one has:

$$
\sup _{x \in \mho_{1}}\left|D_{j+1} \cdots D_{m+1} F(x)\right| \leqq C^{m-j+1}(m-j+\beta) !^{d} .
$$

These last two inequalities yield:

$$
\begin{aligned}
& \sup _{\substack{x \in \mathscr{Q}( \\
0 \leqq t \leq \hat{\delta} \\
0 \leq t}} \mid\left(D_{0} \cdots D_{j-1} D_{t}^{\beta} K_{\nu}^{*}\left(D_{j} \alpha\right)\left(D_{j+1} \cdots D_{m+1} g\right)(x, t) !\right. \\
& \quad \leqq C^{i p !+\beta+1}(|p|+\beta) !^{d} .
\end{aligned}
$$

Since $\delta$ was arbitrary and $\mathscr{C}$ was an arbitrary compact set contained in $\boldsymbol{R}_{0}^{n} 4.3$ and 4.4 shows that the first term in 4.1 belongs to $\Gamma^{d}\left(V \times R_{+}\right)$. That $D_{t}^{\beta} D_{x}^{p}\left(K_{\nu}^{* \prime}(1-\alpha) F\right)$ is in $\Gamma^{d}\left(V \times R_{+}\right)$is done in a similar manner.

Proof of Theorem 1.1. Let $\Omega_{1}$ be a relatively compact open subset of $\Omega$ with plane piece of boundary $\omega_{1} \subset \omega$ such that $\bar{\Omega}_{1} \cup \omega_{1} \subset$ $\Omega \cup \omega$. Let $\alpha \in C_{C}^{\infty}\left(\overline{\boldsymbol{R}}_{+}^{n+1}\right)$ be such that $\alpha \equiv 1$ in $\overline{\Omega_{1} \cup \omega_{1}}$ and its support is contained in $\Omega \cup \omega$. Let $u$ be a solution of 1.1. By using $\alpha$ as a cut off function we see that

$$
\begin{aligned}
& P\left(D, D_{t}\right) U=g \quad \text { in } \Omega \cup \omega \\
& Q_{\nu}\left(D, D_{t}\right) U=h_{\nu} \quad \text { in } \omega \quad 1 \leqq \nu \leqq \mu
\end{aligned}
$$

with $g$ in $\Gamma^{d}\left(\Omega_{1} \cup \omega_{1}\right)$, and $h_{\nu} \in \Gamma^{d}\left(\omega_{1}\right)$, and $g$ and $h_{\nu}$ are continuous with compact support in $\Omega \cup \omega, U=\alpha u$.

Consider

$$
K_{0}^{*} g+\sum_{\nu=1}^{\mu} K_{\nu}{ }^{\prime} h_{\nu}
$$

The problem

$$
\begin{aligned}
& P\left(\xi, D_{t}\right) \hat{U}(\xi, t)=\hat{g}(\xi, t) \\
& Q_{\nu}\left(\xi, D_{t}\right) \hat{U}(\xi, 0)=h_{\nu}(\xi) \quad 1 \leqq \nu \leqq \mu
\end{aligned}
$$

for sufficiently large $\xi$ (i.e. $|\xi| \geqq M$ ) has a unique solution given by:

$$
\int_{0}^{\infty} \hat{K}_{0}(\xi, t-s) \hat{g}(\xi, s) d s+\sum_{\nu=1}^{\mu} \hat{K}_{\nu}(\xi, t) \hat{h}_{\nu}(\xi) .
$$

Thus 


$$
\begin{aligned}
\hat{U}(\xi, t)= & \psi(\xi) \hat{U}(\xi, t)+(1-\psi(\xi)) \hat{U}(\xi, t) \\
= & \psi(\xi) \hat{U}(\xi, t)+\int_{0}^{\infty}(1-\psi(\xi)) \hat{K}_{0}(\xi, t-s) \hat{g}(\xi, s) d s \\
& +\sum_{\xi=1}^{\mu}(1-\psi(\xi)) \hat{K}_{2}(\xi, t) \hat{h}_{2}(\xi) .
\end{aligned}
$$

Taking back the Fourier transform and applying the PaleyWiener theorem one gets that

$$
U(x, t)=\beta(x)^{* \prime} U(x, t)+K_{0}^{*} g+\sum_{\nu=1}^{\prime \prime} K_{\nu}(x, t)^{* \prime} h_{\nu},
$$

with $\beta(x)$ analytic in $\boldsymbol{R}^{n}$, as a solution to 4.5 .

It follows from Lemma 4.1 that the last two terms belong to $\Gamma^{d}\left(\Omega_{1} \cup \omega_{1}\right)$. Since $\beta(x)$ is analytic the convolution $\beta(x)^{*^{\prime}} U(x, t)$ is analytic in $x$ and of class $C^{m}$ with respect to the transversal variable t. Finally since

$$
P\left(D, D_{t}\right) U=D_{t}^{m} U(x, t)+\cdots+\alpha_{m}(D) U(x, t)=g
$$

and $g \in \Gamma^{d}\left(\Omega_{1} \cup \omega_{1}\right)$ it follows that $U \in \Gamma^{d}\left(\Omega_{1} \cup \omega_{1}\right)$. Finally since $\Omega_{1} \cup \omega_{1}$ was arbitrary it follows that

$$
u \in \Gamma^{d}(\Omega \cup \omega) .
$$

\section{REFERENCES}

1. R. A. Artino, Gevrey classes and hypoelliptic boundary value problems, Thesis, Rutgers University, June 1971.

2. _- On semi-elliptic boundary value problems, J. Math. Anal. Appl., 42 3: (1973).

3. Jose Barros-Neto, Kernels associated to general elliptic problems, J. of Funct. Anal.,

3, No. 2, (1969), 172-192.

4. - On Poisson's kernels, Anais da Academia Brasileria de Ciencias, 42, No. 1, (1970), 1-3.

5. - On the existence of fundamental solutions of boundary value problems, Proc. of the Amer. Math. Soc., 24, No. 1, (1970), 75-78.

6. - The parametrix of a regular hypoelliptic boundary value problem, Ann. Scoula Norm Sup. Pisa, 26, Fasc., 1 (1972).

7. - On regular hypoelliptic boundary value problems, J. Math. Anal. Appl., 41 (1973).

8. Lars Hörmander, The regularity of solutions of boundary value problems, Acta Math., 99 (1958), 225-264.

9. — Linear Partial Differential Operators, Springer-Verlag, 1963.

10. L. Schwartz, Théorie des Distributions, (3-e éd.) Hermann, Paris, 1966.

11. A. Seidenberg, A new decision method of elementary algebra, Ann. of Math. (2) 60 (1954), 364-374.

12. Francois Treves, Partial Differential Operators with Constant Coefficients, Gordon and Breach, 1968.

Received March 30, 1972 and in revised form October 27, 1975.

The City College of the City University of New York 



\section{PACIFIC JOURNAL OF MATHEMATICS}

EDITORS

RICHARD ARENS (Managing Editor)

University of California

Los Angeles, California 90024

R. A. BEAUMONT

University of Washington

Seattle, Washington 98105
J. DugundJI

Department of Mathematics

University of Southern California

Los Angeles, California 90007

D. Gilbarg and J. Milgram

Stanford University

Stanford, California 94305

\section{ASSOCIATE EDITORS}

E. F. BECKENBACH

B. H. NeumanN

F. WOLF

K. YosHIDA

\section{SUPPORTING INSTITUTIONS}

UNIVERSITY OF BRITISH COLUMBIA

UNIVERSITY OF SOUTHERN CALIFORNIA

CALIFORNIA INSTITUTE OF TECHNOLOGY

UNIVERSITY OF CALIFORNIA

STANFORD UNIVERSITY

UNIVERSITY OF TOKYO

MONTANA STATE UNIVERSITY

UNIVERSITY OF UTAH

UNIVERSITY OF NEVADA

WASHINGTON STATE UNIVERSITY

NEW MEXICO STATE UNIVERSITY

UNIVERSITY OF WASHINGTON

OREGON STATE UNIVERSITY

UNIVERSITY OF OREGON

OSAKA UNIVERSITY

AMERICAN MATHEMATICAL SOCIETY
NAVAL WEAPONS CENTER

Printed in Japan by International Academic Printing Co., Ltd., Tokyo, Japan 


\section{Pacific Journal of Mathematics}

\section{Vol. 63, No. $1 \quad$ March, 1976}

Ralph Artino, Gevrey classes and hypoelliptic boundary value problems ....... 1

B. Aupetit, Caractérisation spectrale des algèbres de Banach commutatives .... 23

Leon Bernstein, Fundamental units and cycles in the period of real quadratic

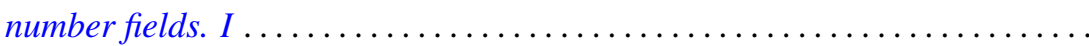

Leon Bernstein, Fundamental units and cycles in the period of real quadratic number fields. II.................................... 63

Robert F. Brown, Fixed points of automorphisms of compact Lie groups ........

Thomas Ashland Chapman, Concordances of noncompact Hilbert cube

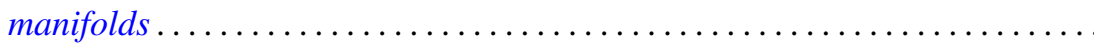

William C. Connett, V and Alan Schwartz, Weak type multipliers for Hankel

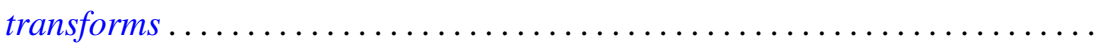

John Wayne Davenport, Multipliers on a Banach algebra with a bounded approximate identity .....................................

Gustave Adam Efroymson, Substitution in Nash functions ................ 137

John Sollion Hsia, Representations by spinor genera ..................

William George Kitto and Daniel Eliot Wulbert, Korovkin approximations in

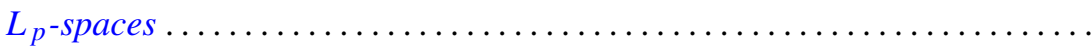

Eric P. Kronstadt, Interpolating sequences for functions satisfying a Lipschitz. condition ...........................................

Gary Douglas Jones and Samuel Murray Rankin, III, Oscillation properties of certain self-adjoint differential equations of the fourth order...

Takaŝi Kusano and Hiroshi Onose, Nonoscillation theorems for differential

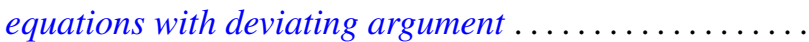

David C. Lantz, Preservation of local properties and chain conditions in commutative group rings. ...

Charles W. Neville, Banach spaces with a restricted Hahn-Banach extension property....

Norman Oler, Spaces of discrete subsets of a locally compact group ...

Robert Olin, Functional relationships between a subnormal operator and its minimal normal extension.

Thomas Thornton Read, Bounds and quantitative comparison theorems for nonoscillatory second order differential equations ...... .

Robert Horace Redfield, Archimedean and basic elements in completely distributive lattice-ordered groups...

Jeffery William Sanders, Weighted Sidon sets

Aaron R. Todd, Continuous linear images of pseudo-complete linear topological spaces.

J. Jerry Uhl, Jr., Norm attaining operators on $L^{1}[0,1]$ and the Radon-Nikodým property.

William Jennings Wickless, Abelian groups in which every endomorphism is a left multiplication. 